\title{
Cytodiagnosis of Dimorphic Fungi with Disseminated Infection in a HIV Positive Patient - A Diagnostic Dilemma
}

\author{
Chitresh Yadav ${ }^{1}$, Nandini N. M. ${ }^{1}$, Shruti M. K. ${ }^{1}$, Sowmya G. S. ${ }^{2}$, M. R. Shivaprakash ${ }^{3}$, \\ G. V. Manjunath ${ }^{1}$ \\ ${ }^{1}$ Department of pathology, faculty JSS Medical College \& hospital, constituent of JSS University, Mysore, India \\ ${ }^{2}$ Department of Microbiology, faculty JSS Medical College \& hospital, constituent of JSS University, Mysore, India \\ ${ }^{3}$ Medical mycology division, Post Graduate Institute of Medical Education and Research, Chandigarh, India
}

Email address:

chitresh2yadav@gmail.com (C. Yadav),nanda_dr05@yahoo.com (Nandini N. M.)

\section{To cite this article:}

Chitresh Yadav, Nandini N. M., Shruti M. K., Sowmya G. S., M. R. Shivaprakash, G. V. Manjunath. Cytodiagnsis of Dimorphic Fungi with Disseminated Infection in a HIV Positive Patient - A Diagnostic Dilemma. Science Journal of Clinical Medicine. Special Issue: Latest Different Concepts of Gynaecology. Vol. 4, No. 4-1, 2015, pp. 19-22. doi: 10.11648/j.sjcm.s.2015040401.15

\begin{abstract}
Systemic mycosis caused by fungi (or dimorphic fungi) such as Histoplasma, emmonsia, candida \& penicillium has emerged as an important opportunistic fungal infection in immunocompromised patient in South- East Asia. Several cases of opportunistic fungal infection has been reported but cytological diagnosis of disseminated (dimorphic) fungal infection has been reported in very few cases. Cytological diagnosis of these infections depending on the morphological characteristics has always been a challenge to practising cytologists. A case of rare disseminated fungal infection reported in a 45 year old seropositive male who is noncompliant on ART presented with ulcer over palate, fever, weight loss, occasional per rectal bleeding. On examination having bilateral cervical lymphadenopathy and hepatomegaly. On investigation- anaemia, high ESR, deranged liver function test, CD4 count 16/cumm, USG showed hepatomegaly, underwent FNAC cervical lymphnodes and guided FNAC of liver, which showed these organisms, but no pathogens were found with classical microbiological procedure. Patient was treated with itraconazole and amphotericine and showed improvement. This case illustrate the utility of FNAC in early diagnosis \& management of rare infection \& it also highlight the combined role of molecular technique and FNAC as an interdisciplinary approach for management of patient.
\end{abstract}

Keywords: Dimorphic Fungal Infection, FNAC Lymph Node, Seropositive

\section{Introduction}

Systemic infections caused by fungi constitute a major public health problem in many parts of the world, both in developed and developing countries ${ }^{1,2}$.

Fungi are extremely fit for survival as evidenced by their ubiquity in nature. However, of the estimated several hundred thousand species of fungi, fewer than 150-200 were considered to be pathogens of humans. However, in recent years, fungi are flourishing in man. The number of fungi causing systemic disease is growing and the number of systemic diseases caused by fungi is increasing. Up to $7 \%$ patients dying in teaching hospitals have invasive aspergillosis ${ }^{3,4,5}$. Candida spp. accounts for $8-15 \%$ of nosocomial blood stream infections and fourth most common isolate of patients of intensive care unit ${ }^{6}$. Specific patient groups have very high frequencies of fungal infections: $15 \%$ of allogenic hemopoietic stem-cell transplant recipients have a fungal infection ${ }^{7}$, about $20 \%$ of lung transplant recipients are colonized and infected ${ }^{8}$, about $60 \%$ and $20 \%$ of AIDS patients have Pneumocystis carinii ( jiroveci) pneumonia or esophageal candidiasis, respectively ${ }^{9}$ cryptococcal meningitis is present in $30 \%$ of people with AIDS in Africa and southeast Asia ${ }^{10}$ and Penicillium marneffei infections are present in about $30 \%$ of people with AIDS in south-east Asia $^{11}$. With the emergence of AIDS. Sometimes, it is not just a single fungus, but rather a combination of fungi i.e. species under Pneumocystis, Candida, Cryptococcus, Histoplasma, Coccidioides, Aspergillus, and zygomycetes, which may produce concomitant and/or successive opportunistic systemic fungal infections ${ }^{3}$. 


\section{Case Report}

A 45 year old seropositive male, positive for HIV-1 on irregular ART since last 10months presented to ENT outpatient department of JSS Hospital, Mysore with 15 days history of pain while eating, fever, generalized itching \& weight loss since 2 months. On examination he was febrile(101 f), having an ulcer of the soft palate, bilateral cervical lymphadenopathy, macular lesions over skin of hand and back with mild hepatomegaly for which he was referred to dermatology department, he was admitted for treatment of fever.

On investigation he was anaemic (Haemoglobin- 9.4 $\mathrm{gm} / \mathrm{dl}$ ), complete haemogram showed Pancytopenia, deranged liver function profile, Total bilirubin 6.15, Direct3.03, AST-238 IU/L, ALT- 164IU/L, ALP- 5545 IU/L, Total protein- $6.0 \mathrm{gm} / \mathrm{dl}$, albumin- $3.0 \mathrm{gm} / \mathrm{dl}$, A:G- 1.0, normal coagulation profile, CD4 count- 16/cumm, positive for HIV type-1, Negative for- HIV, HBsAg, Anti HCV \&VDRL, ESR-120 mm/hour, renal function test \& blood sugar were with in normal limit, urine \& stool examination were also with in normal limit, $\mathrm{KOH}$ from nail clipping \& from ulcer showed no fungal element.

FNAC cervical lymph node \& Ultra sound guided FNAC of liver was performed, Cell block was made from the liver aspirate \& material was sent for culture and showed the following findings.

FNAC Cervical lymph nodes- Aspirate was scantily cellular \& showed macrophages with multiple intracellular \& extracellular yeast forms, with ill-defined granulomas. Yeast forms showed basophilic centre \& surrounding halo. (Fig $1,2,3,4)$

USG Guided FNAC Liver- Hepatocytes \& liver macrophages showed similar intracellular yeast forms.

Cell block (Liver aspirate): Showed basophilic capsulated organism in liver macrophages. (Fig 7)

Special stains: PAS \& GMS- positive (fig 5,6)

Based on above findings diagnosis of disseminated opportunistic fungal infection with organism morphologically resembling yeast forms of Histoplasma was made.

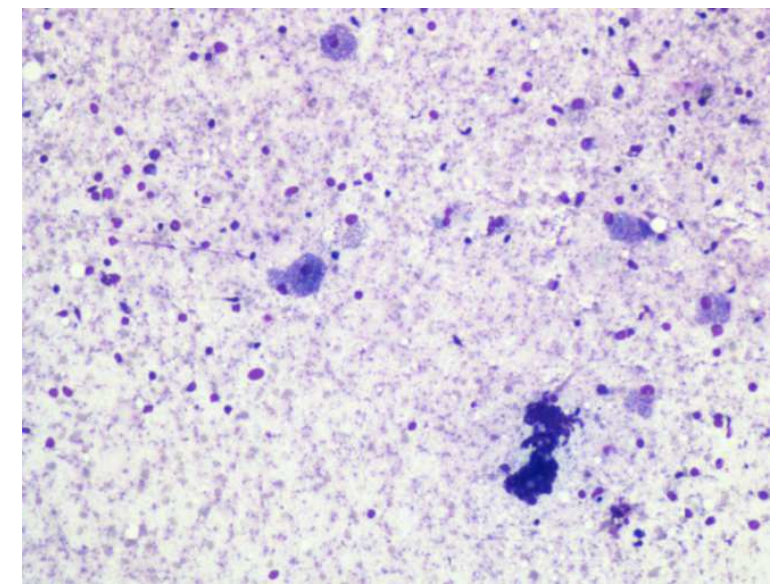

Fig. 1. FNAC Cervical lymph node shows scanty material with macrophages $\&$ few lymphocytes $(H \& E, x 100)$

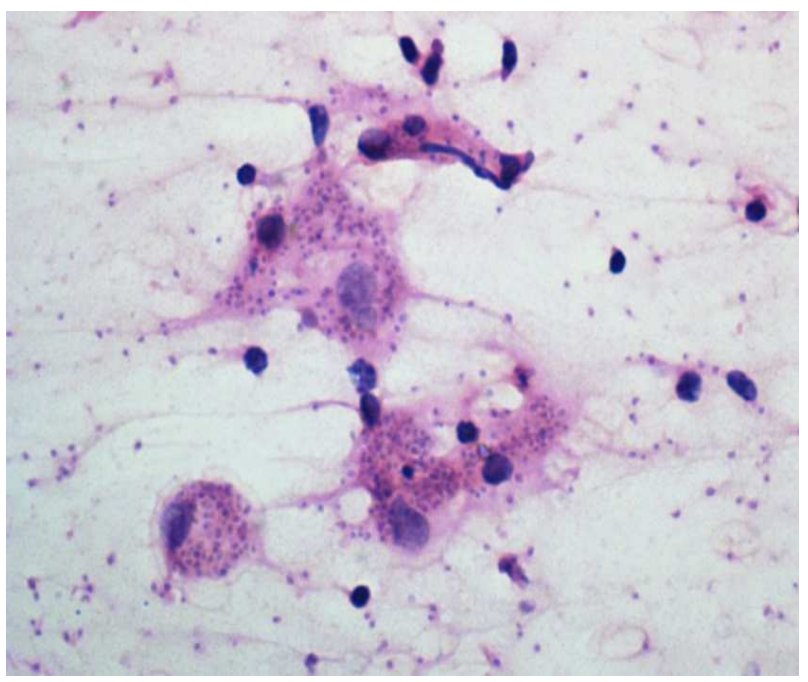

Fig. 2. FNAC Cervical lymph node shows macrophages with intracellular \& extracellular organisms (H\&E stain,x400).

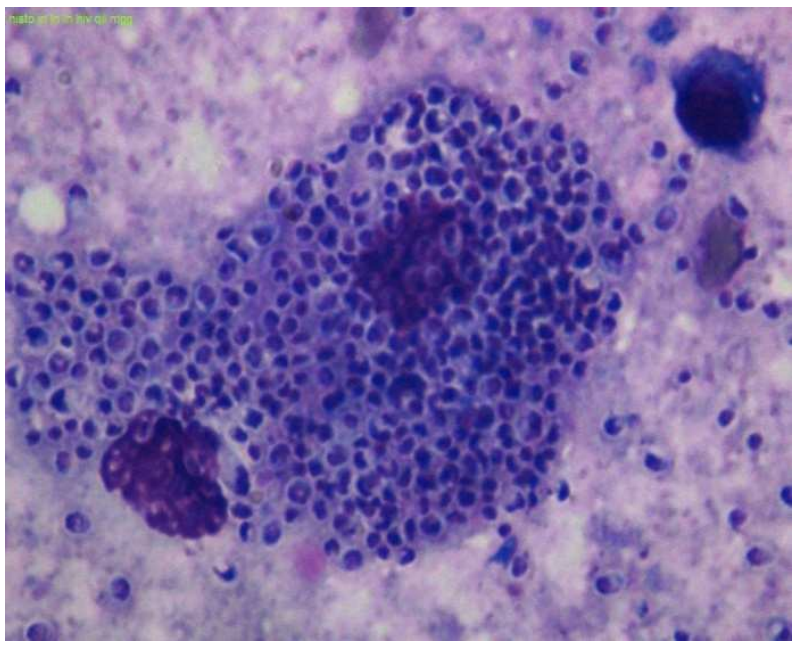

Fig. 3. FNAC Cervical lymph node showing macrophage with both intracellular \& extracellular yeast forms (MGG, x1000).

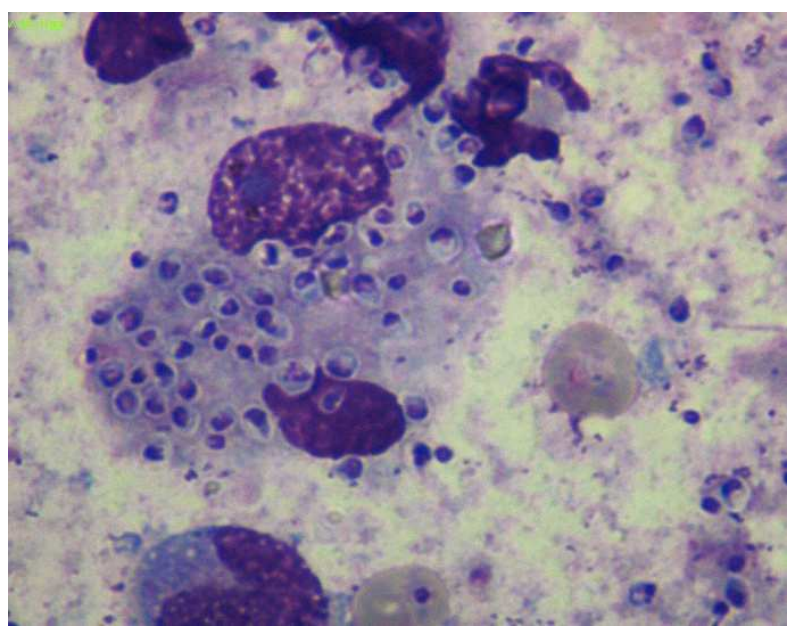

Fig. 4. FNAC Cervical lymph node showing macrophage with both intracellular \& extracellular yeast forms with well basophilic centre \& surrounding halo (MGG, x1000). 


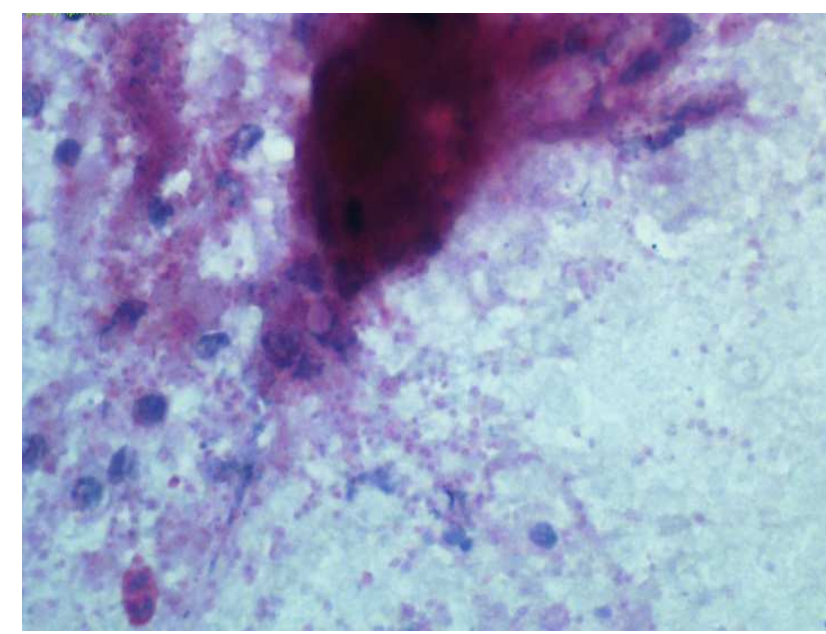

Fig. 5. FNAC Cervical lymph node showing both intracellular \& extracellular PAS Positive organism (x400).

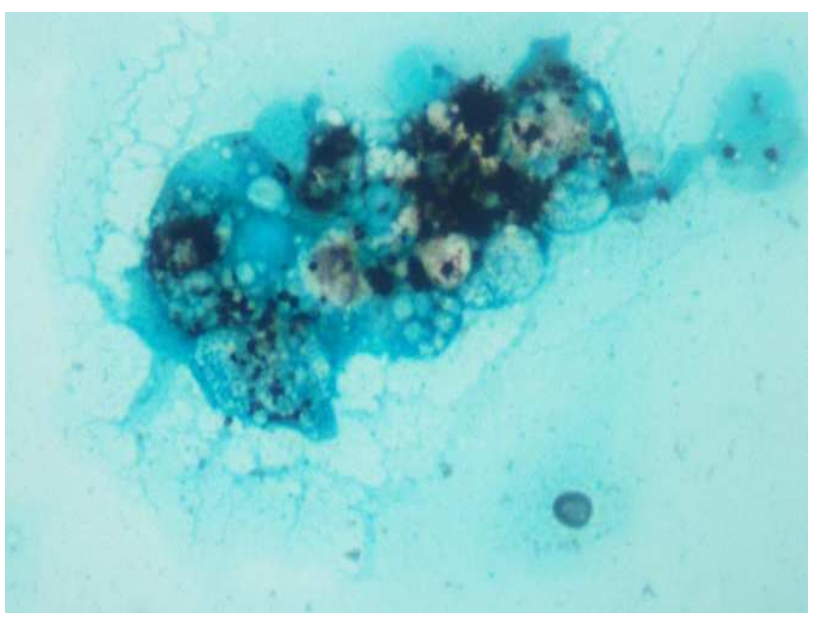

Fig. 6. FNAC cervical lymph node showing GMS positivity(x400).

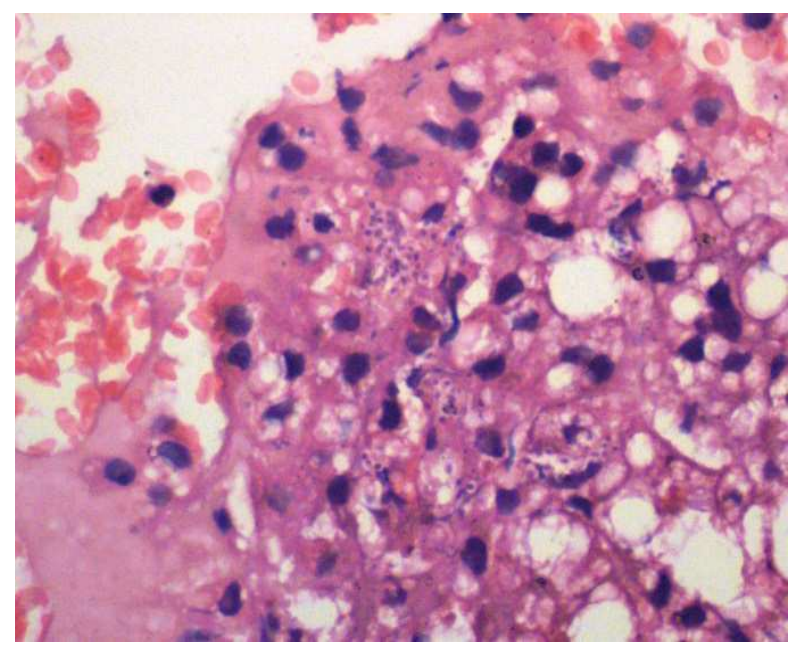

Fig. 7. Cell Block (FNAC Liver) Liver macrophages showing phagocytized intracellular organisms ( $H \& E$ stain, $x 400)$.

Patient responded to amphotericine-B injections \& other symptomatic treatment with regularization of his ART treatment.

Since the material aspirated was less so culture showed no growth.
Specimen was send to PGIMER Chandigarh for species specific PCR which was negative for Histoplasma, candida, penicillium \& emmonsia.

\section{Discussion}

Lymph nodes in patients with HIV infection may contain fungi, atypical mycobacteria or many other microorganism without the usual indicators of these infections. HIV lymphadenitis may be associated with a spectrum of changes, ranging from florid lymphoid hyperplasia to marked lymphoid depletion. In the depletion phase, aspirates often have sparse follicular center cells, immunoblasts, and tingible body macrophages but high numbers of plasma cells ${ }^{12,13}$.

Differential diagnosis on cytology in seropositive patient: Since all the given below organisms can present with cervical lymphadenopathy, can involve liver, skin \& known to cause disseminated infection, it is important to know cytomorphological features and special stain appearance of these organisms to differentiate them \& coming to a proper conclusion.

- H. capsulatum- Small, uninucleate dimorphic fungus measuring 2-4 $\mu \mathrm{m}$ in diameter, found predominantly intracellularly or can be extracellular and is usually present in clusters inside the cytoplasm of macrophages, with a light area surrounding the organism (pseudocapsule)14,15,16.

- Cryptococci- Round capsulated organisms with a thick capsule \& show positive capsular staining on Mayer's mucicarmine but negative staining with India ink preparation, do not form aggregates \& show narrow budding 15,16 .

- P. marneffei- Spherical-to-oval yeast like organism found in macrophages, reproduces by schizogony (fission) with the formation of a prominent transverse septum15.

- Leishmania will not take PAS stain and is distinguished by a nucleus and bar-shaped kinetoplast lying often right angle to nucleus within each amastigote14,15.

- Emmonsia. spp- Three known species- Emmonsia Crescence(most common in asia), Parva(Africa), Pasteuriana) Shows intra \& extra cellular oval-crescent conidia with central halo with in a distinct thick trilaminar thick walled adiaspore, which are positive for GMS \& PAS stain in a background of granulomatous process 17.

\section{Conclusion}

This case illustrates the utility of FNAC in early diagnosis and management of rare fungal infections. Opportunistic infections can present with variable clinical spectrum, which should be considered while reporting on immunocompromised patients. Cytologists should be aware of cytomorphological features of opportunistic mycotic infections. Based on morphological characteristics organisms can be identified on cytology \& confirmed by ancillary 
techniques. An interdisciplinary approach for diagnosis is almost always necessary.

\section{References}

[1] Sunita singh, Sonia chhabra, Ruchi goyal et.al. Cytodiagnosis of histoplasma case report from two patient with variable clinical presentation. Diagnostic Cytopathology. 09/2011; DOI: $10.1002 /$ dc. 21803 .

[2] Manoj harnalika, Vidya kharkar, Uday khopkar. Disseminated cutaneous histoplasmosis in an immunocompetent adult. Indian journalof dermatology. 2012; 57(3); 206-209; DOI: 10.4103/0019-514.96194.

[3] Chakrabarti A, Shivaprakash M R. Microbiology of systemic fungal infections. J Postgrad Med 2005;51, Suppl S1:16-20.

[4] Groll AH, Shah PM, Mentzel C,Schneider M, Just-Nuebling G, Huebner K. Trends in the postmortem epidemiology of fungal infections at a University hospital. J Infect 1996; 33:23-32. [PUBMED].

[5] Vogesar M, Hass A, Aust D, Ruckdeschel G. Postmortem analysis of invasive aspergillosis in a tertiary care hospital. Eur J Clin Microbiol Inf Dis 1997;16:1-6.

[6] Pfaller MA, Diekema DJ. Role of sentinel surveillance of candidemia: Trends in species distribution and antifungal susceptibility. J Clin Microbiol 2002;40:3551-7.

[7] Ribaud P, Chastang C, Latge JP, et al . Outcome and prognostic factors of invasive aspergillosis after allogenic bone marrow transplantation. Clin Infect Dis 1999;28:322-30.

[8] Denning DW. Invasive aspergillosis. Clin Infect Dis 1998;26:781-805.
[9] Moore RD, Chaisson RE. Natural history of opportunistic disease in an HIV infected urban clinical cohort. Ann Intern Med 1996;124:633-42.

[10] Grant AD, Djomand G, DeCock KM. Natural history and spectrum of disease in adults with HIV/AIDS in Africa. AIDS 1997;11:S43-54.

[11] Supparatpinyo K, Khamwan C, Baosoung V, Nelson KE, Sirisanthana T. Disseminated Penicillium marneffei infection in southeast Asia. Lancet 1994;344:110-3.

[12] Mills, Stacey E: Sternberg's Diagnostic Surgical Pathology, 5th Edition. 2010; page no 962-963.

[13] Koss, Leopold G, Melamed, Myron R: Koss' Diagnostic Cytology and Its Histopathologic Bases, 5th Edition. 2006; page no 1194.

[14] Sankha koley, Rjesh kumar mandal, Kalyan khan et.al. Disseminated Cutaneous Histoplasmosis, an Initial Manifestation of HIV, Diagnosed with Fine Needle Aspiration Cytology. Indian J Dermatol. 2014 Mar-Apr; 59(2): 182-185.

[15] Gupta N, Arora SK, Rajwanshi A, Nijhawan R, Srinivasan R. Histoplasmosis: Cytodiagnosis and review of literature with special emphasis on differential diagnosis on cytomorphology. Cytopathology 2010;21:240-4.

[16] Nancy A. Rihana, Manasa Kandula, Ana Velez et.al. Histoplasmosis presenting as granulomatous hepatitis: case report and review of the literature. Hindawi publishing corporation. Vol 2014; Article ID 879535; 4 pages; DOI: org10.1155/2014/879535.

[17] Yanyu Sun, Tawfiq Bbhuiya, Tarun Wasil et.al. Fine needle aspiration of pulmonary adiaspiromycosis-A case report. Acta cytol. 2007; 51; 217-221. 